\title{
Dopaminergic Contributions to Hippocampal Pathophysiology in Schizophrenia: A Computational Study
}

\author{
Peter J Siekmeier, ${ }^{*, 1,2}$ and David P vanMaanen ${ }^{1,2}$ \\ 'Laboratory for Computational Neuroscience, McLean Hospital, Belmont, MA, USA; ${ }^{2}$ Harvard Medical School, Boston, MA, USA
}

\begin{abstract}
Since the original formulation of the dopamine hypothesis, a number of other cellular-level abnormalities-eg, NMDA receptor hypofunction, GABA system dysfunction, neural connectivity disturbances-have been identified in schizophrenia, but the manner in which these potentially interact with hyperdopaminergia to lead to schizophrenic symptomatology remains uncertain. Previously, we created a neuroanatomically detailed, biophysically realistic computational model of hippocampus in the control (unaffected) and schizophrenic conditions, implemented on a 72-processor supercomputer platform. In the current study, we apply the effects of dopamine (DA), dose-dependently, to both models on the basis of an exhaustive review of the neurophysiologic literature on DA's ion channel and synaptic level effects. To index schizophrenic behavior, we use the specific inability of the model to attune to the $40 \mathrm{~Hz}$ (gamma band) frequency, a finding that has been well replicated in the clinical electroencephalography (EEG) and magnetoencephalography literature. In trials using 20 'simulated patients', we find that DA applied to the control model produces modest increases in $40 \mathrm{~Hz}$ activity, similar to experimental studies. However, in the schizophrenic model, increasing DA induces a decrement in $40 \mathrm{~Hz}$ resonance. This modeling work is significant in that it suggests that DA's effects may vary based on the neural substrate on which it acts, and-via simulated EEG recordings_-points to the neurophysiologic mechanisms by which this may occur. We also feel that it makes a methodological contribution, as it exhibits a process by which a large amount of neurobiological data can be integrated to run pharmacologically relevant in silico experiments, using a systems biology approach.

Neuropsychopharmacology (2014) 39, 1713-1721; doi:I0.1038/npp.2014.19; published online 26 February 2014
\end{abstract}

Keywords: dopamine; hippocampus; schizophrenia; computational modeling; gamma band; systems biology

\section{INTRODUCTION}

A large body of literature suggests dopamine (DA) is involved in the genesis of schizophrenic symptoms, but the exact mechanism by which it exerts its effects remains unclear. Much current thinking suggests that schizophrenia may be associated with subcortical hyperdopaminergiabased in large part on a substantial neuroimaging literature indicating increased presynaptic release of $\mathrm{DA}$ in the striatum of schizophrenic patients (Howes et al, 2012) and prefrontal cortex (PFC) hypodopaminergia (Guillin et al, 2007). Moreover, it has been suggested that although hyperdopaminergia may not be the sole cause of the illness, it may be involved in the exacerbation of symptoms or at least some subset of them. A clearer understanding of these questions would shed light on the neurobiological basis of the illness and, in turn, potentially point the way to more effective treatments.

\footnotetext{
*Correspondence: Dr PJ Siekmeier, Laboratory for Computational Neuroscience, McLean Hospital, I I 5 Mill Street, deMarneffe \#239, Belmont, MA 02478, USA, Tel: + I 617855 3588, Fax: + I 617855 4231,E-mail: psiekmeier@mclean.harvard.edu Received 24 June 2013; revised 20 January 2014; accepted 21 January 20।4; accepted article preview online 28 January 2014
}

Understandably, recent attention has focused not only on the specific DA abnormalities seen in schizophrenia, but also on how these may lead to particular clinical manifestations of the illness. DA is probably best regarded not simply as 'excitatory' or 'inhibitory'. Rather, recent work has suggested that it may alter the processing of information in a region-specific manner (eg, Santesso et al, 2009). Dopaminergic innervation originates primarily in the ventral tegmental area (VTA) and nearby substantia nigra, and from here projects widely. The primary DA pathways are the mesocortical, which projects from VTA to prefrontal cortex, the mesolimbic, which projects from VTA to the hippocampus, amygdala, and nucleus accumbens, and nigrostriatal, which projects from substantia nigra to the striatum. Much recent research has focused on the subcortical nigrostriatal system as well as the mesocortical projections to PFC and other frontal cortex areas. For example, work by Winterer (2006) has suggested that electrophysiologic activity in the PFC of schizophrenic patients may be characterized by higher levels of cortical response variability (or 'noise'), and that increased DA levels may correlate with decreased PFC noise, an effect possibly mediated via DA's action on excitatory NMDA synapses and inhibitory GABA synapses. Consistent with this view, computational models examining the importance of DA in PFC function in the control (Durstewitz et al, 2000) 
and schizophrenic (Komek et al, 2012) conditions have been described.

Relatively less attention has been paid to the mesolimbic DA projections terminating in the hippocampus (Miyake et al, 2011). However, the neuroanatomic literature has shown that there are abundant VTA-to-hippocampal projections (eg, Gasbarri et al, 1997, 1994), and that the human hippocampus is rich in DA receptors (De Keyser, 1993). In the current study, we focus on DA's modulatory effects on the hippocampus, a brain area that has been implicated in the pathogenesis of schizophrenia, particularly the genesis of positive symptoms. The hippocampus is known to be important in the storage and recall of memories and the formation of associations. The mechanism by which this is achieved is not known with certainty, but it is likely that synchrony in the gamma frequency band $(\sim 40 \mathrm{~Hz})$ is of central importance to healthy hippocampal functioning (Colgin and Moser, 2010), and is disrupted in schizophrenia (Gandal et al, 2012; Lisman et al, 2008).

Attempts to understand DA's effects on hippocampal functioning in a detailed, mechanistic way-and how this may go awry in schizophrenia-are made complicated by two considerations. First, it has become clear that DA affects neural processing not through a single electrophysiologic effect but through a broad range of activities on synaptic and ion-channel functioning (Beaulieu and Gainetdinov, 2011). Second, recent research has made abundantly clear that other neurotransmitters-eg, the glutamatergic and GABAergic systems-are abnormal in the hippocampus in schizophrenia. In addition, aberrant connectivity (Hoffman and McGlashan, 2003) has also been seen. A priori, it is not clear how DA deficiencies would interact with these abnormalities.

In this paper, we address these questions using a systems biology approach. We used a previously developed, biophysically detailed computational hippocampal model (Siekmeier and Vanmaanen, 2013) implemented on a 72-processor supercomputer, and took a specific quantitative deficit in the $40 \mathrm{~Hz}$ auditory steady state response (ASSR) as index of schizophrenic behavior. DA effects are applied via alterations in a constellation of synaptic and ion channel effects, in a receptor-mediated manner, both to an unaffected (control) and to a schizophrenic model. We show in the schizophrenic model that increasing DA levels lead to a decrement in gamma band response, and that in the control model similar DA levels show a modest enhancement of gamma band activity (similar to experimental work). This work may have implications for the development of novel agents to treat schizophrenia.

\section{METHODS}

\section{Background and Model Construction}

In the ASSR task, subjects receive auditory stimuli at various frequencies, and cortical response is measured via electroencephalography (EEG) or magnetoencephalography; typically, frequencies present in the response signal are determined via Fourier transform or wavelet analysis. Stimulation protocols include a range of frequencies (eg, 20,30 , and $40 \mathrm{~Hz}$ ), and generally control subjects show a strong cortical response at the stimulated frequency. Schizophrenic patients, however, show a unique inability to attune to gamma band $(\sim 40 \mathrm{~Hz})$ stimulation-multiple studies have shown that schizophrenic patients' $40 \mathrm{~Hz}$ response to the $40 \mathrm{~Hz}$ drive is markedly lower than that of controls, whereas response at other frequencies (eg, 20 and $30 \mathrm{~Hz}$ ) is comparable to those of controls (Gandal et al, 2012). A recent review of the literature on oscillatory abnormalities in schizophrenia (Uhlhaas and Singer, 2010) found that across all experimental protocols-ie, those looking at spontaneous activity, as well as those using induced, evoked, and ASSR paradigms - the most robust effect was found in patients in the gamma band ASSR task. Thus, this may be tapping into a key neural deficit in schizophrenia and may represent a sensitive biomarker of the illness (Javitt et al, 2008).

The computational model used in this paper is based on the one detailed in our previous published work. Briefly, the model consists of 160 pyramidal cells and 80 interneurons of three subtypes (basket cells, chandelier cells, and calretininpositive cells). The pyramidal cell model is based on the 64 compartment model of Traub, et al (1994); the interneuron model is derived from the 51 compartment model of Traub and Miles (1995). They both include realistic dendritic arbors and incorporate $\mathrm{Na}^{+}, \mathrm{Ca}^{2+}, \mathrm{K}^{+}{ }_{\mathrm{DR}}, \mathrm{K}^{+}{ }_{\mathrm{AHP}}, \mathrm{K}^{+}{ }_{\mathrm{C}}$, and $\mathrm{K}^{+}{ }_{\mathrm{A}}$ channels distributed along the somato-dendritic axis; intracellular $\mathrm{Ca}^{2+}$ concentration is explicitly represented. The connectivity of the model was arrived at based on an exhaustive review of the neuroanatomic literature on projection patterns of hippocampal pyramidal neurons and interneuron subtypes, and was designed to emulate area CA1. It also includes simulated DA receptors.

In the study described here, we drive the model - that is, we deliver input stimulation to the constituent computational neurons-at 20,30, and $40 \mathrm{~Hz}$. This is meant to recreate the auditory stimuli used in the experimental human studies using the ASSR task, at the frequencies most commonly used. For each run, a simulated EEG, calculated based on Nunez (1981), is written to file. We use two distinct network models throughout our study, corresponding to the control and schizophrenic cases. The manner in which we created these models is described in Siekmeier and Vanmaanen (2013). Briefly, we tuned the control model to show similar 20,30 , and $40 \mathrm{~Hz}$ behaviors to the controls in experimental studies. We arrived at the schizophrenic model by (1) exhaustively reviewing the relevant literature on NMDA function, dendritic spine density, and GABA system integrity in schizophrenic hippocampus and, based on the ranges of abnormalities found, (2) searching the parameter space of putative schizophrenic neural abnormalities, using the response to the ASSR task as our outcome metric. Compared with the control model, the schizophrenic model has a $30 \%$ decrease in NMDA conductance and dendritic spine density.

The model is implemented using the General Neural Simulation System neural modeling software,version 2.3, with MPI programs written in $\mathrm{C}++$ for parameter searches. The hardware platform used is a 72-processor dedicated Beowulf Cluster running under Linux (Red Hat Enterprise Linux 5.0).

\section{Operationalization of Dopaminergic Effects}

At baseline in humans, extracellular DA levels are said to be around $10-20 \mathrm{nM}$, and with activity intrasynaptic 
concentrations can reach the micromolar range (Grace, 2011). Direct measures of tissue-level increases of DA in schizophrenic patients are, of course, very difficult to obtain. Indirect measures involving $\left({ }^{11} \mathrm{C}\right)$ raclopride binding (Abi-Dargham et al, 2000; Laruelle et al, 1999b) or CSF samples (Gattaz et al, 1983) have tended to show modest DA increases in patients. To ensure that any possible effect is captured, we modeled DA increases ranging over several orders of magnitude, from 0 to $1 \mathrm{mM}$.

DA receptors can broadly be divided into D1-like receptors (D1 and D5) and D2-like receptors (D2, D3, and D4). Human postmortem studies that have done direct comparisons have shown that D1 receptors outnumber D2 receptors in the hippocampus, and indicated that this difference is considerable. For example, Hall et al (1988) assessed D1 vs D2 binding in postmortem human brain using homogeneous (that is, not subfield specific) samples from the hippocampus, and found average D1 concentrations of $1.4 \mathrm{pmol} / \mathrm{g}$ vs negligible concentrations of D2 receptors. De Keyser (1993), in a detailed review of the literature, estimated that the ratio of D1 to D2 receptors in the human hippocampus is $\sim 11$ to 1 . Studies that have done subfield-specific analyses have found that in area CA1 this difference is even more marked. For example, Court et al (1998) showed that in CA1 D1 density was $30.03 \mathrm{fmol} /$ $\mathrm{mg}$ tissue, whereas D2 density was $0.18 \mathrm{fmol} / \mathrm{mg}$ tissue, a 17-fold difference. Gangarossa et al (2012) looked at DA binding in a cell type-specific manner (ie, pyramidal cells $v s$ interneurons). In CA1, they found no D2 binding in either cell type to speak of. Therefore, in the current study, we focus on D1-type receptor-mediated effects.

There is an extensive literature on the manner in which DA exerts neurophysiologic effects by altering ion channel and synaptic conductances. For example, this research has shown that DA significantly decreases conductance of the $\mathrm{K}_{\mathrm{AHP}}$ (Pedarzani and Storm, 1995) and $\mathrm{K}_{\mathrm{C}}$ (Satake et al, 2008) channels, increases conductance at AMPA synapses (Yang, 2000), and has small or negligible effects at the $K_{D R}$ and $\mathrm{K}_{\mathrm{A}}$ channels (Dong and White, 2003). The experimental literature used to arrive at all parameter values for DA implementation is described in detail Supplementary Methods. Our methodology for introducing DA effects into the model in a concentration-dependent manner is detailed below.

We assumed that the fraction of DA receptors bound is a function of DA concentration, and its dissociation constant at the DA receptor, $K_{\mathrm{i}}$, is represented as follows (Kenakin, 1997):

$$
P=\frac{[D A]}{[D A]+K_{i}}
$$

Here, $P$ is the percentage of DA receptors bound. We take $K_{\mathrm{i}}$ to be 4.3 (Tiberi and Caron, 1994) (expressing the dissociation constant as the negative exponent of the concentration, in molar terms, at which $50 \%$ of receptors are occupied).

We assumed that the ultimate neurophysiologic effect (eg, increase or decrease in ion-channel conductance, expressed as $E_{\mathrm{A}}$ below) was a linear function of DA receptor occupancy. That is,

$$
E_{A}=E_{\max } x P
$$

Table I $E_{\max }$ Values

\begin{tabular}{lll}
\hline Channel & $\boldsymbol{E}_{\mathbf{M}} \mathbf{( \% )}$ & Source \\
\hline $\mathrm{NMDA}$ & -33.6 & Castro et al (1999) \\
$\mathrm{AMPA}$ & +5.0 & Yang (2000) \\
$\mathrm{GABA}_{A}$ & -23.0 & Liu et al (2000) \\
$\mathrm{Na}^{+}$ & -22.2 & Cantrell et al (1997) \\
$K_{A H P}^{+}$ & -35.9 & Pedarzani and Storm (1995) \\
$\mathrm{Ca}^{+}+$ & -20.0 & Surmeier et al (1995) \\
$K_{D R}^{+}$ & 0.0 & Dong and White (2003) \\
$K_{A}^{+}$ & -24.5 & Dong and White (2003) \\
$K_{C}^{+}$ & -48.1 & Sataki et al (2008) \\
\hline
\end{tabular}

Conductance change at full dopamine receptor occupancy for model ion channels and synaptic conductances. Experimental studies from which values were derived are given. Experiments generally used saturating concentrations, and in these cases determination of $E_{\max }$ was straightforward. In those cases in which non-saturating concentrations were used (eg, Castro et al, 1999; Dong and White, 2003), we calculated $E_{\max }$ using Equations I and 2, in text. Full D | occupancy was also seen to increase the decay time constant $\left(\tau_{2}\right)$ of the AMPA channel by about 50\% (Yang, 2000); this was also included in the model, in a manner analogous to the conductance effects.

The proportionality constant $E_{\max }$ (Kenakin, 1997) is different for each distinct ion channel or synapse. These values were derived from experimental studies that quantified the change in channel conductance when tissue was exposed to particular concentrations of DA or DA agonist. $E_{\max }$ values and the experimental studies from which they were derived are shown in Table 1 .

Given the purposes of our study, we have chosen to focus on the relatively acute effects of DA, as outlined above. We acknowledge that this may be dissimilar from the effects of chronic DA elevation (ie, months to years), which may be associated with changes in receptor density, etc.

\section{Simulated Experimental Protocol}

We drove the control (non-schizophrenic) hippocampal model at 20,30, and $40 \mathrm{~Hz}$, and the schizophrenic model in the baseline (no DA) condition, and at increasing simulated concentrations of DA in 10 gradations. At each simulated concentration, we calculated the percentage of DA receptors occupied, using Equation (1). Then, based on this, we calculated the percentage change in conductance in each of the constituent ion channels of the model, using Equation (2) and the values of Table 1. Thus, for example, at a DA concentration of $67 \mu \mathrm{M}, 57 \%$ of DA receptors are bound (from Equation (1)). At this level, $\mathrm{Na}^{2+}$ conductance is decreased by $57 \% \times 0.222=0.126, \mathrm{Ca}^{2}+$ conductance is decreased by $57 \% \times 0.20=0.114$, etc. At the maximum simulated concentration $(1 \mathrm{mM})$, there is complete receptor saturation and all conductance changes in Table 1 apply. An identical series of trials were performed for the schizophrenic model. These two virtual experiments correspond to clinical experiments in which control and schizophrenic patients were subjected to the ASSR task in the presence and absence of dopaminergic agents (Albrecht et al, 2013; Komek et al, 2012). 

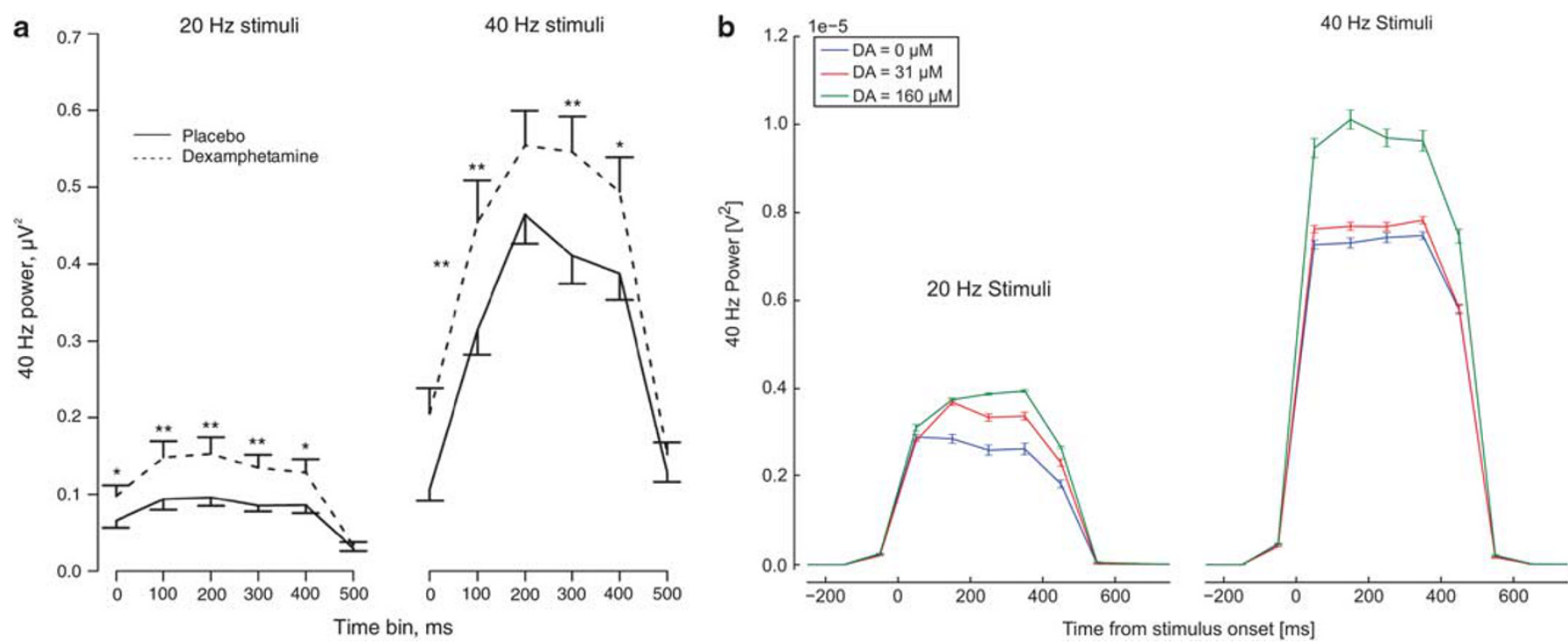

Figure I Auditory steady state response (ASSR) task in control subjects: experimental vs computational results. (a) Findings of Albrecht et al (20I3), in which subjects were exposed to 20 and $40 \mathrm{~Hz}$ stimuli. Dexamphetamine was used to induce hyperdopaminergia. Bars indicate standard error (b) Computational model outputs for analogous task. Because it is not entirely clear how dexamphetamine dosage used by Albrecht et al (0.45 mg/kg) would translate quantitatively to serum level, we used a range of DA concentrations. To simulate the clinical experimental task, we stimulated with background noise for $500 \mathrm{~ms}$, then drove the model (as described in the text) for $500 \mathrm{~ms}$, then delivered background noise. Response power at $40 \mathrm{~Hz}$ is shown on the $y$-axis; the $x$-axis indicates time from onset of this stimulus. Data are binned at $100 \mathrm{~ms}$. To emulate clinical experiment as closely as possible, all simulations were run with 20 'simulated subjects'. To generate a simulated subject, we re-seeded the simulation's random number generator and re-created the hippocampal model. This resulted in a model with a statistically identical pattern of connectivity (defined by the probability with which a given cell type projects to another cell type), but a different specific cell-to-cell pattern of connectivity. Bars indicate standard error.

\section{RESULTS}

We first needed to confirm the validity of the control model by quantitatively comparing its behavior with that observed in clinical studies. Albrecht et al (2013) studied 44 unaffected (non-schizophrenic) subjects using the ASSR task, with 20 and $40 \mathrm{~Hz}$ stimulation. In one trial subjects were given dexamphetamine, to induce hyperdopaminergia, and in the other they received placebo. They used an experimental protocol in which stimuli $(20$ or $40 \mathrm{~Hz}$ click trains) were presented for $500 \mathrm{~ms}$, with $500 \mathrm{~ms}$ interstimulus intervals, and they found that when treated with dexamphetamine subjects showed a significantly greater $40 \mathrm{~Hz}$ response to $40 \mathrm{~Hz}$ stimulation and also significantly greater $40 \mathrm{~Hz}$ response to $20 \mathrm{~Hz}$ stimuli (Figure 1a). When we carried out their experiment computationally, we achieved similar results (Figure $1 \mathrm{~b}$ ).

Next, we performed the ASSR task in the control and schizophrenic models at increasing levels of DA, as detailed in Methods. Significantly, increasing levels of simulated DA affected these two models in a dissimilar manner. In the schizophrenic case, it suppressed the $40 \mathrm{~Hz}$ ASSR response at intermediate concentrations. As indicated in Figure 2, this effect was not simply a result of suppression of oscillatory behaviors generally. In the control model, it has an opposite effect, modestly increasing the $40 \mathrm{~Hz}$ response at these concentrations. At higher DA concentrations, both control and schizophrenic models showed increased $40 \mathrm{~Hz}$ response to increased simulated receptor occupancy. Thus, as measured by gamma frequency response, the control model showed a monotonic response to increasing dopaminergic drive, and the schizophrenic model showed a U-shaped response (Figure 2 and Table 2).
In order to investigate whether or not a particular parameter is more or less important than others in the instantiation of the concentration-dependent DA effect, we performed a 'sensitivity analysis', as described in Supplementary Results. It was seen that GABA synaptic conductance and the AMPA decay time constant $\left(\tau_{2}\right)$ were particularly important mediators of DA's effect on oscillatory activity. Model behavior with these effects removed, alone and in combination, is shown as Supplementary Figures S1, S2, and S3.

In an additional set of trials, D1-mediated effects on synaptic plasticity at NMDA synapses were included-this was implemented via intracellular $\mathrm{Ca}^{2+}$ concentration effects, using the methodology described in Siekmeier and Vanmaanen (2013). Resultant patterns of oscillatory activity were very similar to those of Figure 2 (data not shown). This is understandable, given the fact that this is an activitydependent phenomenon, and the period of synchronous stimulation is relatively brief.

An interesting-and counterintuitive-behavior of the schizophrenic model is its tendency to show an increase in $20 \mathrm{~Hz}$ response to $40 \mathrm{~Hz}$ drive (dashed line in Figure 2), while showing a decrease in $40 \mathrm{~Hz}$ response, as DA concentration increased. This was not present in the control model. In an attempt to understand the mechanistic basis of this behavior, we examined a simulated EEG trace and associated power spectrum for a representative schizophrenic and control patient. The manner in which these changed as simulated DA concentrations are increased is shown in Figure 3.

Of note, the decrease in $40 \mathrm{~Hz}$ response at intermediate DA concentrations seen in the schizophrenic model does not result from an across-the-board decrement in the amplitude of this frequency. Rather, the EEG trace reveals that every other peak is suppressed, thus creating decreased 
a

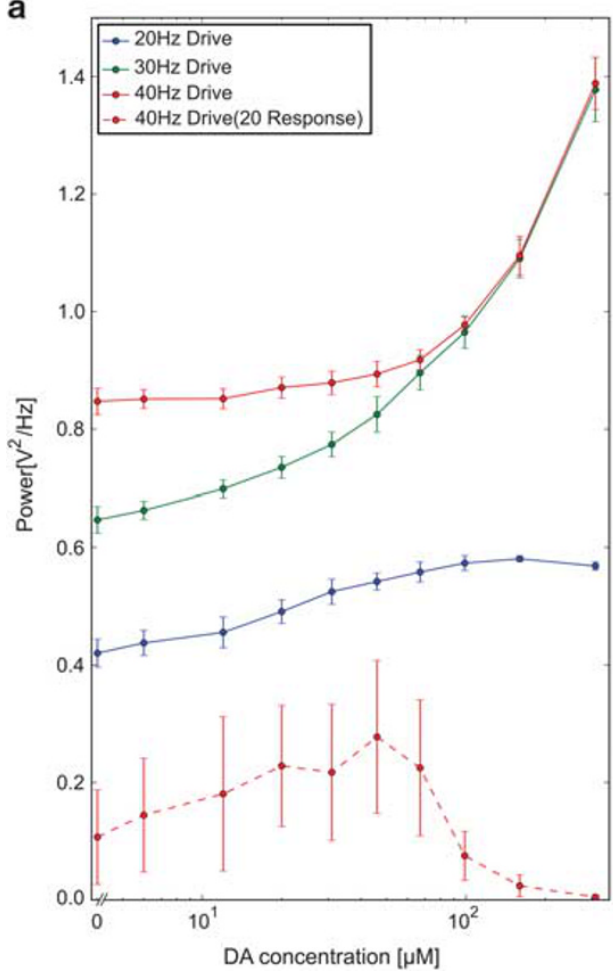

b

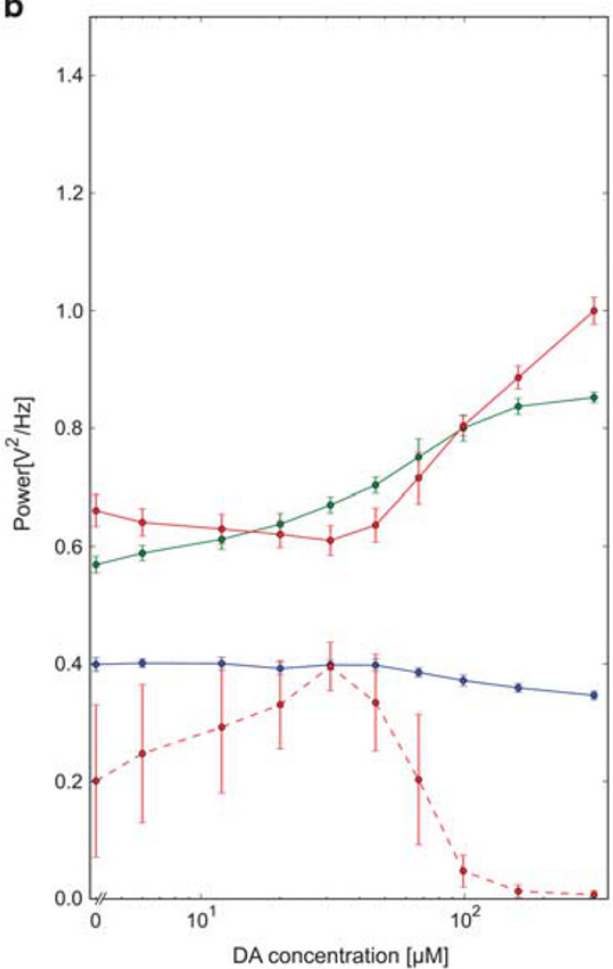

Figure 2 Response of control (a) and schizophrenic (b) model at increasing levels of simulated dopamine. As indicated in the legend, $20 \mathrm{~Hz}$ response power to $20 \mathrm{~Hz}$ drive, $30 \mathrm{~Hz}$ response to $30 \mathrm{~Hz}$ drive, $40 \mathrm{~Hz}$ response to $40 \mathrm{~Hz}$ drive, and $20 \mathrm{~Hz}$ response to $40 \mathrm{~Hz}$ drive are shown. Eleven DA concentrations, ranging from 0 to I $\mathrm{mM}(=1000 \mu \mathrm{M})$, were performed. It is evident that the effect of DA in suppressing gamma activity in the schizophrenic case (b) is specific to that frequency: at the DA concentration associated with the greatest decrease in $40 \mathrm{~Hz}$ response $(3 \mathrm{I} \mu \mathrm{M})$, $20 \mathrm{~Hz}$ response is not statistically significantly different from baseline $(P=0.84)$, and $30 \mathrm{~Hz}$ response is actually greater than baseline $(P<0.000 \mathrm{I})$. At I000 $\mu \mathrm{M}$, the model showed a behavior consistent with the graphic trends shown (data not shown). For each trial, 20 'simulated patients' (see Figure I caption) were used. Error bars indicate standard deviation. P-values are given in Table 2 and text.

Table $240 \mathrm{~Hz}$ Response of Control and Schizophrenic Models at Various Levels of Simulated Dopamine

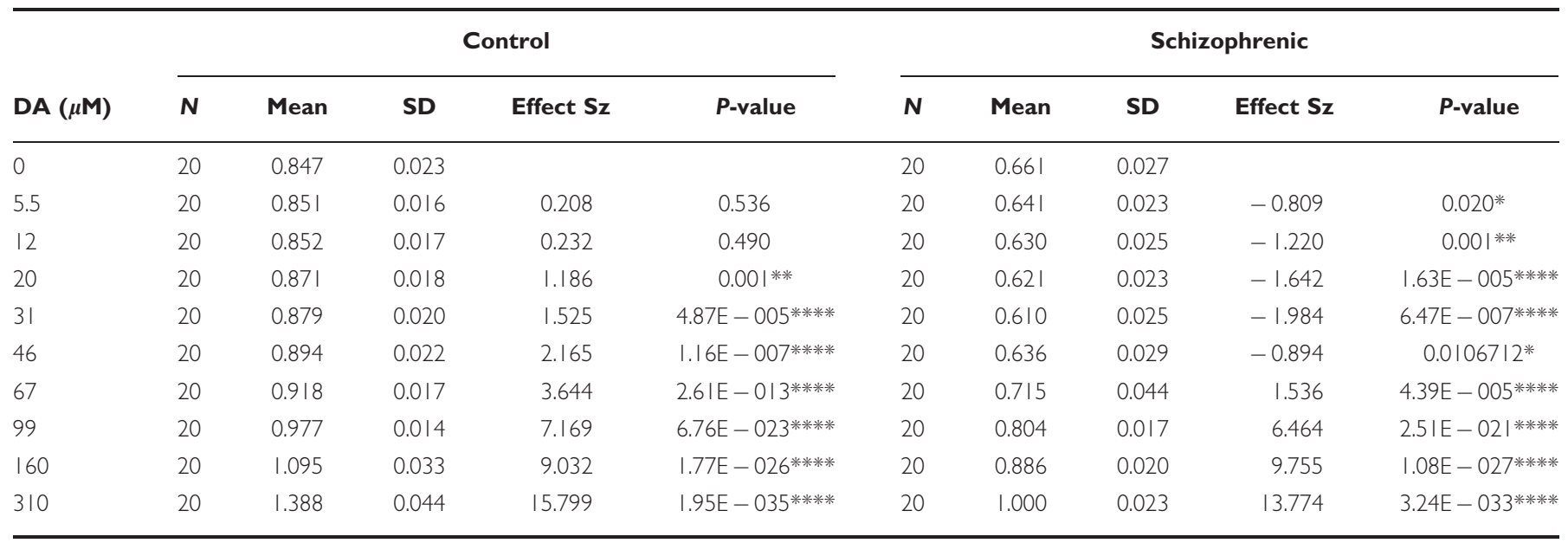

Values for $40 \mathrm{~Hz}$ response to $40 \mathrm{~Hz}$ drive are given. Effect size (Cohen's d) represents difference from baseline (0 dopamine) condition. Note opposite effects at intermediate dopamine levels in control and schizophrenic models. $* p<0.05, * * * 0.005, * * * p<0.0005, * * * * p<0.00005$.

power at $40 \mathrm{~Hz}$, and the emergence of a stronger $20 \mathrm{~Hz}$ signal.

\section{DISCUSSION}

A substantial research literature suggests that DA is involved in the genesis of schizophrenic symptoms, but the mechanism by which this occurs is not entirely clear. Moreover, since the original formulation of the DA hypothesis, large numbers of abnormalities-in other neurotransmitter systems, and in brain connectivityhave been identified in the illness. This computational modeling study offers a possible way of integrating these findings: it suggests how DA's neurophysiological and 

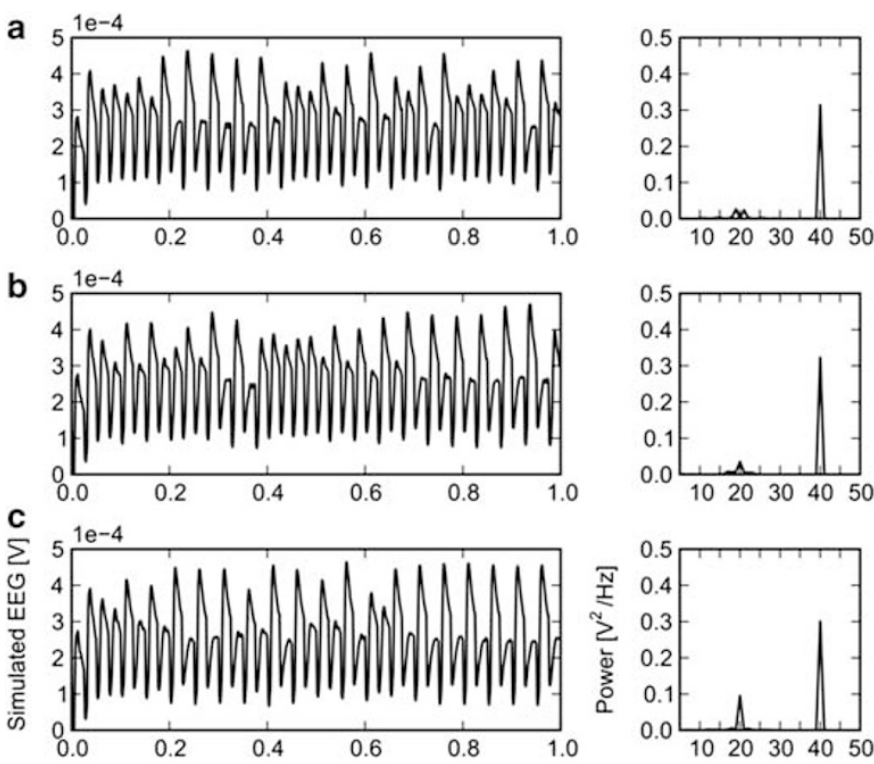

d

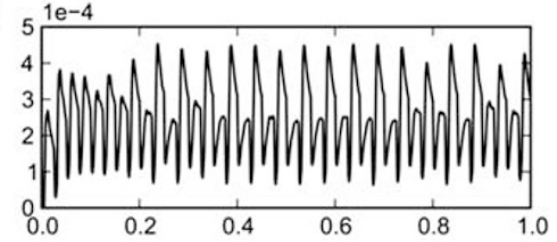

e
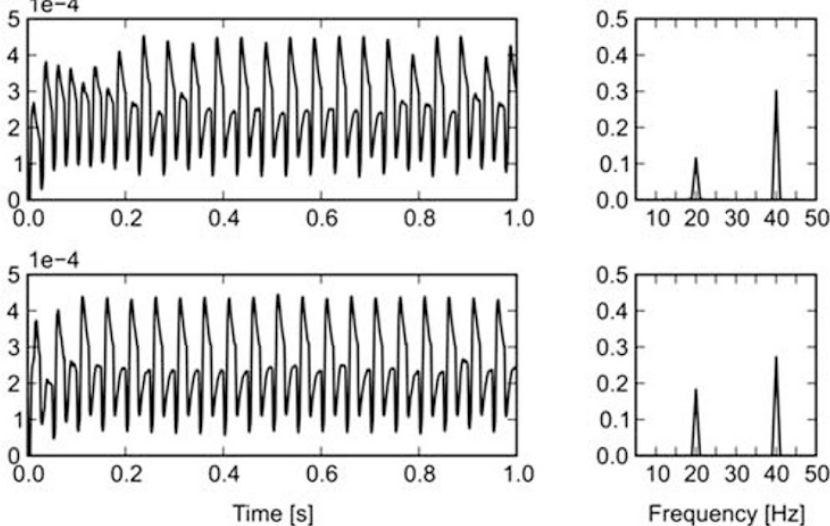

f
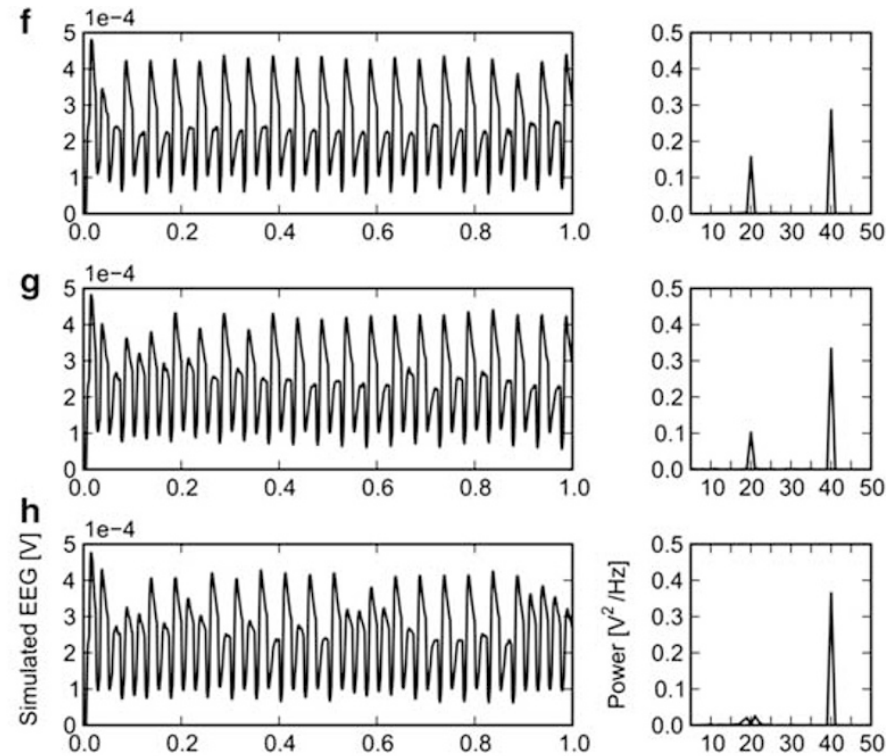

i $51 \mathrm{e}^{-4}$
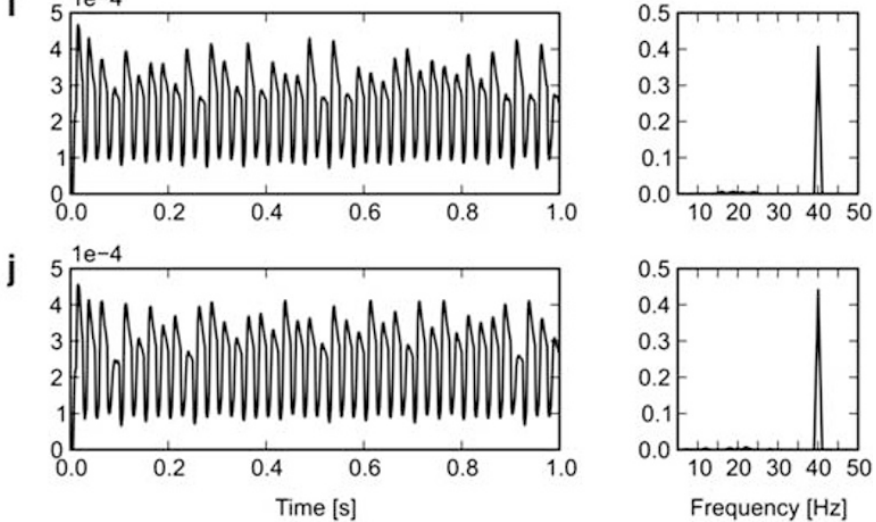

Figure 3 EEG response of a representative schizophrenic case to increasing levels of simulated dopamine. (a) Baseline ( 0 dopamine) condition for a sample simulated patient; EEG response to $40 \mathrm{~Hz}$ stimulation, and power spectrum of response, are shown. In panels (b) through (j), the simulated dopamine level is successively increased, with concentrations corresponding to those of Figure 2 and Table 2, to a maximum of 3 I $0 \mu$ M. Only I s of a I.5 s run is shown so that the 'beat-skipping' behavior may be clearly seen.

neurocognitive effects may vary, depending on the neural substrate on which it acts. Specifically, in a hippocampus afflicted with the NMDA dysfunction and connectivity disturbances thought to be associated with schizophrenia, hyperdopaminergia appears to worsen to gamma band synchrony deficit that has been seen in the illness, whereas in an unaffected hippocampus this effect appears to be absent. Also, it potentially explains the episodic nature of schizophrenia and supports the view, articulated in the clinical research literature, that DA may act as 'the wind of the psychotic fire' (Laruelle and Abi-Dargham, 1999a).

\section{Comparison with Experimental Findings}

One clue to DA's role in the emergence of schizophrenic symptoms comes from clinical studies in which dopaminergic agents (eg, amphetamine) are administered to control subjects and schizophrenics. Our modeling results are consistent with these findings, in that these studies have generally shown that such agents exacerbate psychotic symptoms in patient groups, but, with acute administration, do not tend to create psychotic symptoms de novo in controls. In a review of 36 studies, Lieberman et al (1987) found that $\sim 40 \%$ of schizophrenic patients showed a worsening of psychotic symptoms after acute administration of psychostimulants $v s 2 \%$ of controls $(P<0.0001)$.

There are a few clinical studies that have examined the effect of dopaminergic manipulation on the ASSR task in control (Albrecht et al, 2013) as well as schizophrenic subjects (Komek et al, 2012). As mentioned above, our work replicated the findings of Albrecht et al on control subjects (Figure 1). In Komek et al's study of the effect of D-amphetamine administration on $40 \mathrm{~Hz}$ ASSR in schizophrenic patients, they included 12 schizophrenic patients as well as 12 controls and found that patients showed a modest increase in $40 \mathrm{~Hz}$ response $(P<0.05)$ when they received amphetamine at a dosage of $0.5 \mathrm{mg} / \mathrm{kg}$. It is somewhat difficult to interpret the results of their study in light of ours because, for practical reasons, only a single dose was given as opposed to a range of doses. It is possible that the dose administered led to a high degree of receptor occupancy, and therefore an enhancement of gamma response (as 
indicated at higher DA concentrations in Figure 1b). This group also found that the controls showed a decrease in gamma band ASSR in response to amphetamine challenge. One possible reason for this discrepancy from our findings is that these researchers focused on PFC-rather than hippocampus. These two brain areas may differ in DAmodulated ASSR response.

Another clinical study whose findings are consistent with those of our computational study is that of Barr et al (2011). This work showed that repetitive transcranial magnetic stimulation delivered to the dorsolateral PFC while subjects were performing an $\mathrm{N}$-back working memory task elicited a reduction in gamma power in schizophrenic patients, but resulted in an enhancement in gamma in controls. This is highly pertinent, as in vivo microdialysis experiments in non-human primates have indicated that a significant increase in DA levels exists in PFC during the performance of working memory tasks (Watanabe et al, 1997).

A potentially significant confound in interpreting all of these clinical studies is the impact of medications. Although some have found no statistically significant relationship between ASSR and antipsychotic dosage in schizophrenic patients (Spencer et al, 2008), others (Hong et al, 2004a) found that patients treated with atypical antipsychotics showed greater $40 \mathrm{~Hz}$ ASSR than both controls and those treated with typical antipsychotics. EEG readings in these studies were taken at the fronto-central electrodes, so they may not be directly translatable to our hippocampal model. However, they do raise the very important point that because all currently used antipsychotics have activity on the DA system, going forward it would be important to specifically consider the nature of these effects in ASSR studies.

\section{Mechanistic Implications}

Neurophysiologically, our study suggests that the decreased gamma band response to $40 \mathrm{~Hz}$ stimulation observed under hyperdopaminergia may arise from a 'beat-skipping' phenomenon-that is, a $40 \mathrm{~Hz}$ response is converted to a mixed-mode 40 and $20 \mathrm{~Hz}$ response via suppression of every other peak of the phasic response. This mechanism was also shown to be operative in the schizophrenic response in the computational studies of Vierling-Claassen et al (2008) as well as Siekmeier and Vanmaanen (2013). Significantly, both of these studies instantiated schizophrenogenic neuropathologies that were dissimilar from those of the current model. Vierling-Claassen et al implemented an increased inhibitory time constant $\left(\tau_{2}\right)$ on chandelier cell projections to pyramidal cells, and Siekmeier and vanMaanen modeled a joint reduction in NMDA conductance and dendritic spine density. This suggests that this particular neurophysiologic signature may be a 'final common pathway', which may be worthy of investigation as a potentially sensitive biomarker for schizophrenic symptoms.

This, of course, is not meant to suggest that 'beat skipping' is the only means by which gamma and beta oscillations may jointly arise in neural tissue. Computational modeling work by Traub et al (1999) investigating the phenomenon of the gamma-to-beta transition that has been observed in hippocampal slice preparations as well as human ERP studies (Hong et al, 2004b) have suggested that the synchronization of gamma and the synchronization of beta may rely on distinct mechanisms, and ones different from the mechanism we have identified.

\section{Pathophysiologic and Treatment Implications}

Many studies have shown that patients experiencing acute psychotic episodes had significantly elevated indices of synaptic DA transmission in the striatum (eg, Howes et al, 2007), indicating that DA levels here may fluctuate with the phase of the illness. Our work suggests similar effects may be at play in the mesolimbic pathway-in particular, those branches projecting to the hippocampus. This points to the manner in which hyperdopaminergia may interact with hippocampal neuropathology to produce symptoms, which can wax and wane over time. Specifically, our model suggests that increasing dopaminergic stimulation affects hippocampal function in a U-shaped manner in schizophrenia (as shown in Figure 2). This should be contrasted to the theorized effects of DA in other brain areas, such as PFC. Here, it is felt that intermediate levels of DA are associated with optimal functioning - that is, functionality appears to follow an inverted U-shaped curve. Modeling work has suggested that appropriate DA levels-that is, levels that are neither too low or too high-are crucial in stabilizing working memory representations (Durstewitz et al, 2000), working memory being a central functional role of PFC. Komek et al (2012) present a computational model of PFC showing a similar phenomenon for gamma band oscillations. Although it is not clear how the response curves to DA in various brain areas 'align', the apparent dissimilarities in hippocampus and PFC indicate why past attempts at developing antipsychotics that have focused on global manipulation of DA activity as indicated by receptor binding may not have been entirely successful.

The modeling described here presents the possibility of a drug development approach in which the 'target' is not a receptor or a single neurobiologic entity, but rather a system-level neurophysiologic behavior associated with schizophrenia. As the authors and others (Geerts et al, 2013) have demonstrated in previous work, models of this kind can be used to suggest novel sets of cellular level interactions that may be ameliorative. This could be a uniquely powerful approach for clarifying the neuropathology underlying schizophrenia and pointing the way to fundamentally new treatments.

\section{FUNDING AND DISCLOSURE}

PJS's work has been funded by the NIMH (MH072771). In addition, he has received compensation for his work as the Web Editor of The Archives of General Psychiatry/JAMA Psychiatry. DPV declares no conflict of interest.

\section{REFERENCES}

Abi-Dargham A, Rodenhiser J, Printz D, Zea-Ponce Y, Gil R, Kegeles LS et al (2000). Increased baseline occupancy of D2 receptors by dopamine in schizophrenia. Proc Natl Acad Sci USA 97: 8104-8109.

Albrecht MA, Price G, Lee J, Iyyalol R, Martin-Iverson MT (2013). Dexamphetamine selectively increases $40 \mathrm{~Hz}$ auditory steady 
state response power to target and nontarget stimuli in healthy humans. J Psychiatry Neurosci 38: 24-32.

Barr MS, Farzan F, Arenovich T, Chen R, Fitzgerald PB, Daskalakis ZJ (2011). The effect of repetitive transcranial magnetic stimulation on gamma oscillatory activity in schizophrenia. PLoS One 6: e22627.

Beaulieu JM, Gainetdinov RR (2011). The physiology, signaling, and pharmacology of dopamine receptors. Pharmacol Rev 63: 182-217. Cantrell A, Smith R, Goldin A, Scheuer T, Catterall W (1997). Dopaminergic modulation of sodium current in hippocampal neurons via cAMAP-dependent phosphorylation of specific sites in the sodium channel alpha subunit. J Neurosci 17: 7330-7338.

Castro NG, de Mello MC, de Mello FG, Aracava Y (1999). Direct inhibition of the N-methyl-D-aspartate receptor channel by dopamine and (+)-SKF38393. Br J Pharmacol 126: 1847-1855.

Colgin LL, Moser EI (2010). Gamma oscillations in the hippocampus. Physiology 25: 319-329.

Court JA, Lloyd S, Thomas N, Piggott MA, Marshall EF, Morris CM et al (1998). Dopamine and nicotinic receptor binding and the levels of dopamine and homovanillic acid in human brain related to tobacco use. Neuroscience 87: 63-78.

De Keyser J (1993). Subtypes and localization of dopamine receptors in human brain. Neurochem Int 22: 83-93.

Dong Y, White FJ (2003). Dopamine D1-class receptors selectively modulate a slowly inactivating potassium current in rat medial prefrontal cortex pyramidal neurons. J Neurosci 23: 2686-2695.

Durstewitz D, Seamans JK, Sejnowski TJ (2000). Dopaminemediated stabilization of delay-period activity in a network model of prefrontal cortex. J Neurophysiol 83: 1733-1750.

Gandal MJ, Edgar JC, Klook K, Siegel SJ (2012). Gamma synchrony: towards a translational biomarker for the treatment-resistant symptoms of schizophrenia. Neuropharmacology 62: 1504-1518.

Gangarossa G, Longueville S, De Bundel D, Perroy J, Herve D, Girault JA et al (2012). Characterization of dopamine D1 and D2 receptor-expressing neurons in the mouse hippocampus. Hippocampus 22: 2199-2207.

Gasbarri A, Sulli A, Packard MG (1997). The dopaminergic mesencephalic projections to the hippocampal formation in the rat. Prog Neuropsychopharmacol Biol Psychiatry 21: 1-22.

Gasbarri A, Verney C, Innocenzi R, Campana E, Pacitti C (1994). Mesolimbic dopaminergic neurons innervating the hippocampal formation in the rat: a combined retrograde tracing and immunohistochemical study. Brain Res 668: 71-79.

Gattaz WF, Riederer P, Reynolds GP, Gattaz D, Beckmann H (1983). Dopamine and noradrenalin in the cerebrospinal fluid of schizophrenic patients. Psychiatry Res 8: 243-250.

Geerts H, Roberts P, Spiros A (2013). A quantitative system pharmacology computer model for cognitive deficits in schizophenia. CPT: Pharmacometrics Syst Pharmacol 2: e36.

Grace AA. Personal communication, (2011).

Guillin O, Abi-Dargham A, Laruelle M (2007). Neurobiology of dopamine in schizophrenia. Int Rev Neurobiol 78: 1-39.

Hall H, Farde L, Sedvall G (1988). Human dopamine receptor subtypes-in vitro binding analysis using 3H-SCH 23390 and 3H-raclopride. J Neural Transm 73: 7-21.

Hoffman RE, McGlashan TH (2003). Schizophrenia: from psychodynamic to neurodynamic theories. In: Sadock JS, Sadock VA (eds). Kaplan and Sadock's Synopsis of Psychiatry. 9th edn (Vol 1) (Lippincott Williams and Wilkins: Philadelphia, PA.

Hong LE, Summerfelt A, McMahon R, Adami H, Francis G, Elliott A et al (2004a). Evoked gamma band synchronization and the liability for schizophrenia. Schizophr Res 70: 293-302.

Hong LE, Summerfelt A, McMahon RP, Thaker GK, Buchanan RW (2004b). Gamma/beta oscillation and sensory gating deficit in schizophrenia. Neuroreport 15: 155-159.

Howes OD, Kambeitz J, Kim E, Stahl D, Slifstein M, Abi-Dargham A et al (2012). The nature of dopamine dysfunction in schizophrenia and what this means for treatment. Arch Gen Psychiatry 69: 776-786.
Howes OD, Montgomery AJ, Asselin MC, Murray RM, Grasby PM, McGuire PK (2007). Molecular imaging studies of the striatal dopaminergic system in psychosis and predictions for the prodromal phase of psychosis. Br J Psychiatry Suppl 51: s13-s18.

Javitt D, Spencer K, Thaker G, Winterer G, Hajos M (2008). Neurophysiological biomarkers for drug development in schizophrenia. Nat Rev Drug Discov 7: 68-83.

Kenakin T (1997). Pharmacologic Analysis of Drug-Receptor Interaction. Lippincott-Raven: New York.

Komek K, Bard Ermentrout G, Walker CP, Cho RY (2012). Dopamine and gamma band synchrony in schizophreniainsights from computational and empirical studies. Eur J Neurosci 36: 2146-2155.

Laruelle M, Abi-Dargham A (1999). Dopamine as the wind of the psychotic fire: new evidence from brain imaging studies. J Psychopharmacol 13: 358-371.

Laruelle M, Abi-Dargham A, Gil R, Kegeles L, Innis R (1999). Increased dopamine transmission in schizophrenia: relationship to illness phases. Biol Psychiatry 46: 56-72.

Lieberman JA, Kane JM, Alvir J (1987). Provocative tests with psychostimulant drugs in schizophrenia. Psychopharmacology 91: 415-433.

Lisman J, Coyle J, Green R, Javitt D, Benes F, Heckers S et al (2008). Circuit-based framework for understanding neurotransmitter and risk gene interactions in schizophrenia. Trends Neurosci 31: 234-242.

Liu F, Wan Q, Pristupa ZB, Yu X, Wang YT, Niznik HB (2000). Direct protein-protein coupling enables cross-talk between dopamine D5 and gamma-aminobutyric acid A receptors. Nature 403: 274-280.

Miyake N, Thompson J, Skinbjerg M, Abi-Dargham A (2011). Presynaptic dopamine in schizophrenia. CNS Neurosci Ther 17: 104-109.

Nunez PL (1981). Electric Fields of the Brain: The Neurophysics of EEG. Oxford University Press: New York. 484 pp.

Pedarzani P, Storm JF (1995). Dopamine modulates the slow $\mathrm{Ca}(2+)$ activated $\mathrm{K}+$ current IAHP via cyclic AMP-dependent protein kinase in hippocampal neurons. J Neurophysiol 74: 2749-2753.

Santesso DL, Evins AE, Frank MJ, Schetter EC, Bogdan R, Pizzagalli DA (2009). Single dose of a dopamine agonist impairs reinforcement learning in humans: evidence from event-related potentials and computational modeling of striatal-cortical function. Hum Brain Mapp 30: 1963-1976.

Satake T, Mitani H, Nakagome K, Kaneko K (2008). Individual and additive effects of neuromodulators on the slow components of afterhyperpolarization currents in layer $\mathrm{V}$ pyramidal cells of the rat medial prefrontal cortex. Brain Res 1229: 47-60.

Sataki T, Mitani H, Nakagome K, Kaneko K (2008). Individual and additive effects of neuromodulators on the slow components of afterhyperpolarizaiton currents in layer $\mathrm{V}$ pyramidal cells of the rat medial prefrontal cortex. Brain Res 1229: 47-60.

Siekmeier PJ, Vanmaanen DP (2013). Development of antipsychotic medications with novel mechanisms of action based on computational modeling of hippocampal neuropathology. PLoS One 8: e58607.

Spencer KM, Salisbury DF, Shenton ME, McCarley RW (2008). Gamma-band auditory steady-state responses are imparied in first episode psychosis. Biol Psychiatry 64: 369-375.

Surmeier D, Bargas J, Hemmings H, Nairn A, Greengard P (1995). Modulation of calcium currents by a D1 dopaminergic protein kinase/phosphatase cascade in rat neostriatal neurons. Neuron 14: 385-397.

Tiberi M, Caron MG (1994). High agonist-independent activity is a distinguishing feature of the dopamine D1B receptor subtype. J Biol Chem 269: 27925-27931.

Traub RD, Jefferys JG, Miles R, Whittington MA, Toth K (1994). A branching dendritic model of a rodent CA3 pyramidal neurone. J Physiol 481: 79-95. 
Traub RD, Miles R (1995). Pyramidal cell-to-inhibitory cell spike transduction explicable by active dendritic conductances in inhibitory cell. J Comput Neurosci 2: 291-298.

Traub RD, Whittington MA, Buhl EH, Jefferys JGR, Faulkiner HJ (1999). On the mechanism of the gamma-beta frequency shift in neuronal oscillations induced in rat hippocampal slices by tetanic stimulation. J Neurosci 19: 1088-1105.

Uhlhaas PJ, Singer W (2010). Abnormal neural oscillations and synchrony in schizophrenia. Nat Rev Neurosci 11: 100-113.

Vierling-Claassen D, Siekmeier P, Stufflebeam S, Kopell N (2008). Modeling GABA alterations in schizophrenia: a link between impaired inhibition and altered gamma and beta range auditory entrainment. J Neurophysiol 99: 2656-2671.

Watanabe M, Kodama T, Hikosaka K (1997). Increase of extracellular dopamine in primate prefrontal cortex during a working memory task. J Neurophysiol 78: 2795-2798.

Winterer G (2006). Cortical microcircuits in schizophrenia-the dopamine hypothesis revisited. Pharmacopsychiatry 39: S68-S71.

Yang SN (2000). Sustained enhancement of AMPA receptor- and NMDA receptor-mediated currents induced by dopamine D1/D5 receptor activation in the hippocampus: an essential role of postsynaptic $\mathrm{Ca} 2+$. Hippocampus 10: 57-63.

Supplementary Information accompanies the paper on the Neuropsychopharmacology website (http://www.nature.com/npp) 